\title{
ACTIVIDADES DEL DEPARTAMENTO
}

PROYECTOS DE INVESTIGACION.

Para el periodo de investigación junio 1983 a ma yo 1984, el Departamento de Matemáticas ha presentado a concurso los siguientes Proyectos de Investigación :

1. Complementos de algunas técnicas de Geoestadistica.

Jefe de este proyecto es don Patricio Rojas More ra Profesor de Matemáticas y Física, Master en Estadistica ( CIENES).

Con este proyecto se pretende:

a) Desarroliar sistemas computacionales que per mitan : Obtener puntos de variograma en el plano en distintas direcciones; obtener estimación de superficies minerali zadas; ajustar modelo matematicos conocidos a los puntos dêl variograma; obtener soluciones a las ecuaciones de Krigeage, para realizar estimaciones, con sus correspondientes varianzas de estimación.

b) Diseñar una tẻcnica para calcular variogramas 
menes mineralizados.

c) Diseñar una têcnica que permita estimar volú

d) Realizar confrontaciones teoricas prácticas.

2. Estimación de la Longitud del Algoritmo de División.

Jefe de este proyecto es don Rololfo Baeza Vega, Profesor de Matemáticas y Física, Master en Matematicas y es tudios completos de doctorado en el Instituto de Matemática Pura e Aplicada (IMPA) Río de Janeiro, Brasil.

En $\mathbb{Z}$, sean $\theta$ el algoritmo minimal y $\psi$ el algo ritmo de la norma, $L_{\theta} y L_{\psi}$ las longitudes respectivas. Se sospecha que $\mathrm{L}_{\theta}<\mathrm{L}_{\psi}$. Uno de los objetivos de este pro yecto es avanzar en la solución de tal conjetura. También se pretende encontrar respecto a problemas tales como:

1) Hallar algunas fómulas asintóticas para $L_{\theta}$

2) Determinar $(u, v) \varepsilon z^{2}$ para que $L_{\theta}(u, v)$ sea máximo.

3) Hallar alguna cola interesante para $L_{\psi}$ donde $\psi$ cs el algoritmo de la norma en $\psi[i]$

3. Diseño de Cursos Universitarios de Matemâticas Introductoria.

Jefe de este proyecto es don Fernando Jorqusa $\mathrm{NO}$ Iina, Profesor de Matemática, Magister en Matemática.

Este proyecto de investigación consiste er ise ñar un modelo de enseñanza para cursos Universitarios je väte mática Introductoria; desarrollar los medios para su imple mentación; determinar un procedimiento de aplicación y experimentarlo en un curso de geometria y finalmente evaiuario.

Los objetivos de este proyecto son :

- Crear en el Departamento de Matematicas las condiciones técnicas y material para el desarrolio de investi 
gaciones en el área de la educación matemática.

- Establecer nexos con especialistas nacionales y extranjeros que trabajen en el área y contribuir, de este modo al desarrollo de la enseñanza de la matematica en el pais.

- Generar por medio de estudios especificos un marco de referencia que permita orientar futuras investiga ciones en el ấrea.

- Estudio de las características y de los antecedentes cognitivos y afectivos de los estudiantes que ingre san a la Universidad del Norte.

- Desarrollo de una metodología de enseñanza aplicable a cursos introductorios de matemáticas universitarias.

- Diseño de un modelo de enseñanza-aprendizaje para la asignatura de geometría analitica.

- Diseño de un curso piloto que incorpore las estrategias y modelos desarrollados.

4. Solución Numérica de la Ecuación de Poisson.

Este proyecto se esta desarrollando a partin de junio de 1982 y participar como investigadores los profesores: Oscar Rojo Jeraldo, Doctor en Matemática y Jefe del Proyecto, Ricardo Soto Montero, Magister en Matematica y Luis Valdivia Reyes, Magister en Matemática.

Se persigue el logro de los siguientes objetivos:

1) Iniciar la investigación en la lînea del Análisis Numêrico y la formación de cuadros de investigadores en el Departamento de Matemáticas.

2) Estudiar, analizar y comparar los métodos de tipo externo para la Solución Numêrica de la Ecuación de Poisson.

3) Implementar programas computacionales.

4) Encontrar eventualmente alguna modificación que haga más eficiente alguno de los métodos estudiados. 
5) Presentar en una publicación final todos los métodos estudiados en forma unificada y con una comparación entre ellos.

Este proyecto ha sido formulado para el año 1983 , en términos de una continuación del que actualmente se desarrolla, (programado inicialmente por dos años) para estudiar especificamente los métodos de tipo interno; manteniendo los mismos objetivos generales.

\section{PROYECTOS DE EXTENSION 1983.}

El Departamento de Matemáticas, ha presentado pa ra el concurso de proyectos de extensión de nuestra Universi dad para el período 1983, los que se detallan a continuación:

1. Segunda Jornada de Matemáticas de la universidad del Norte.

Este proyecto consiste en charlas y cursilios dictados por académicos de nuestro departamento y expositores invitados. Esta jornada se desarrollará durante una semana, en la cual se realizará la segunda escuela de matemáticas y encuentro de matemáticas (organizado y auspiciado por la sociedad de Matemáticas de Chile).

\section{Academias de Matemáticas en la Enseñanza Me-}

\section{dia.}

Esta actividad consiste en la formación de acade mias de matematicas en los establecimientos de enseñanza media de la comuna de Antofagasta, con el propósito de desarro llar con los alumnos, algunos tópicos matemáticos de interés que no se encuentran en los programas oficiales de educacion media.

Se formarán ocho academias, una en cada cciegio bajo la responsabilidad de dos profesores, uno de enseñanza media y otro del Departamento de Matemáticas. Se desiznaráa un jefe de proyecto que tendrá la posibilidad de coordinar las actividades propias de las academias. 
3. Texto de estudio para la asignatura de Geome tria Analitica. (MA-158)

El objetivo de este proyecto es escribir el texto guía para la asignatura de Geometría Analitica (MA-158), para ingenieros civiles, que estara desarrollado por clases y con sus respectivas guís de ejercicios graduados. Se publicará una edición de 300 ejemplares del texto, que serán entregados a Biblioteca Central para su administración.

4. Geometría a distancia a nivel de Educación Básica y Media.

Este proyecto consiste en cursos de geometría pre parados en módulos, dedicados a los profesores de enseñanza básica y media, con el fin de actualizar sus conocimientos de geometría, sin necesidad de asistir a la Universidad. Estos cursos permitirán a los profesores de la zona, contar con una bibliografia sobre los contenidos de geometría incluidos en los nuevos programas vigentes.

\section{Revista de Matemáticas "Proyecciones".}

Consiste en la elaboración de una revista semes tral, nara ser distribuida y/o vendida, entre los académicos universitarios de la región y en establecimientos de enseñan za media. Su contenido está orientado hacia aspectos matemâa ticos de interés general. Tendrá una sección destinada a te mas especializados, pero a nivel de divulgación.

La capacidad de comunicación del gênero humano tiene un sello caracteristico, cuya expresión más relevante es su potencial independencia de los estados de ânimo. Indu dablemente esta capacidad que nos permite el control de la espontaneidad, es también parte de nuestra capacidad de re flexión. Prescindiendo de otros aspectos, de suyo importantes, nos interesa enfatizar la necesidad que para el cultivo del espiritu cientifico, tiene el ejercicio continuo e inten so de la comunicación.

Tan decisivo es este hecho, que se puede asegu rar que el germen, de no pocas de las grandes ideas en la 
ciencia se encuentra en "las comunicaciones" entre cientifi cos. Tambien las controversias que son una forma de comunicación, han contribuido en gran medida al desarrollo del pen samiento.

Asi pues, conservando las debidas proporcicres los integrantes de todo departamento universitario necesitan $y$ con necesidad vital, comunicarse las ideas que a cada instante le sugieren su actividad académica. Por eso toda orga nización de una universidad debe procurar que la comunica ción entre sus funcionarios sea expedita, eficaz, con poder multiplicativo y facilmente extensible a toda la comunidad. El Departamento de Matematicas ha encarado en forma parcial el logro de estos objetivos, mediante reuniones de departamen - bajo el nombre de Coloquios. La temática, como es natural en todo ensayo es y debe ser general hasta que la evolución, consolide o descarte esta metodologia. Consolidarla signifi caría que se ha llegado al nivel de satisfacción de la inquíe tud por la búsqueda de la vía comunicante. Descarta, en cam bio, implicaría que toda la energía creadora de nuestros acă démicos se esta disipando en diálogos esteriles, en comentarios insulsos sobre intracendencia de la cotidianidad.

ESCUELA DE TEMPORADA.

Las autoridades de nuestra Universidad han autori zado la realización de "La Primera Escuela de Temporada", de Departamento de Matemáticas.

Esta escuela está consagrada a la enseñanza de tó picos elementales y avanzados de Matemáticas. Está dirigida a toda la comunidad de la Región, principalmente, al magiste rio, profesionales, estudiantes universitarios y alumnos egr $\bar{e}$ sados de la Enseñanza Media.

Esta escuela cuenta con 18 cursos regulares. Cin co dedicados al perfeccionamiento del magisterio, 5 a profesionales y universitarios y 3 para el alumno egresado de Ense ñana Media que se prepara a ingresar a la Universidad.

La Escuela de Temporada del Departamento de Matemáticas tiene como principal objetivo:

- Brindar perfeccionamiento a los profesores de Enseñanza Básica y Media.

- Orientar a los alumnos egresados de la Enseñan za Media sobre los niveles de los cursos matemáticos de pri - 
mer semestre universitario.

- Actualizar tópicos matemáticos y estadisticos para profesionales que los utilicen.

Los cursos que se van a dictar en ella son :

A. Cursos para el Magisterio.

Curso 1. Geometría Clásica

curso 2. Fundamentos Numéricos y Aplicaciones a

los Enteros.

Curso 3. Matemáticas para Profesores de Enseñan za Básica.

Curso 4. Geometría para Profesores Básicos

Curso 5. Construcciones Geometricas

B. Cursos para Egresados Enseñanza Media.

Curso 6. Introducción al Algebra Superior

Curso 7. Introducción a la Geometría Analitica

Curso 8. Introducción al cálculo

C. Cursos para Profesionales.

Curso 9. Expresión elemental de la Regresión en

Estadistica.

Curso 10. Sistema de Ecuaciones Lineales

una Variable

Curso 11. Métodos de Derivación e Integración de

Curso 12. Geoestadistica

Curso 13. Métodos para Datos Cualitativos. 